\title{
HISTORIA INÉDITA Y ACTUALIDAD DEL ARCHIVO BONPLAND EN EL MUSEO DE FARMACOBOTÁNICA JUAN ANÍBAL DOMÍNGUEZ (FFYB-UBA)
}

\author{
Unpublished history and present of the Bonpland Archive at the Juan Aníbal Domínguez \\ Museum of Pharmacobotany (FFYB-UBA)
}

\author{
LEONARDO M. ANCONATANI'10, MELINA RIABIS ${ }^{1}\left[0\right.$ \& MARCELO L. WAGNER ${ }^{1}[0$
}

\begin{abstract}
Resumen: En el año 1905, Juan Aníbal Domínguez, fundador y director del Museo de Farmacología de la Facultad de Ciencias Médicas de la Universidad de Buenos Aires, y Eugenio Autran, curador del herbario del Museo, se enteran que cursa la carrera de medicina el estudiante Pompeyo Bonpland, nieto del sabio. Ambos académicos saben de la existencia de cierta documentación inédita del primer naturalista, y reciben por medio de ese joven esa valiosa documentación. El Archivo Bonpland es una heterogénea colección de alrededor de 2.050 documentos de variada extensión y diversa complejidad. Comprende desde un período anterior a su llegada al Río de la Plata y finaliza más de 40 años después de su muerte, con cartas recibidas en su domicilio en 1858. Además, hay diarios de viajes con descripciones biológicas novedosas para la ciencia. Desde el punto de vista de su conservación, el archivo se encuentra estable en lo referente de los documentos planos, sin embargo, no ocurre lo mismo con los diarios manuscritos. Por tales motivos se está diseñando un plan de conservación y restauración. Esto último permitirá acceder al archivo a investigadores de la ciencia y aprovechar el gran potencial del Archivo Bonpland.
\end{abstract}

Palabras clave: Conservación, diarios de viaje, documentos históricos, restauración.

Summary: In 1905, Juan Aníbal Domínguez, founder and director of the Pharmacy Museum of the School of Medicine of the University of Buenos Aires, and Eugenio Autran, curator of the Herbarium of the Museum found out that student Pompeyo Bonpland, grandson of the famous Bonpland, was pursuing a medical degree. Both academics knew of the existence of some unpublished documentation of the naturalist, the first, and received this valuable documentation through this young man. The Bonpland Archive is a heterogeneous collection of around 2,050 documents of various lengths and diverse complexity. It ranges from a period prior to Bonpland's arrival at the Río de la Plata and ends more than 40 years after his death, with letters received at his home in 1858. Furthermore, there are travel diaries with biological descriptions new to science. From the point of view of its preservation, the archive is stable in relation to flat documents, however, the same does not occur with manuscript diaries. This is why its conservation and restoration is being planned. The latter will allow science researchers to access the archive and take advantage of the great potential of the Bonpland Archive.

Key words: Conservation, historical documents, restoration, travel diaries.

\section{Introducción}

E1 11 de mayo de 1858 , a los 85 años, murió Aimé Jacques Alexandre Goujaud Bonpland,
Aimé Bonpland, en Santa Ana (actualmente localidad de Bonpland, en el departamento de Paso de los Libres, provincia de Corrientes). Luego de transmitida la noticia, en el mes

${ }^{1}$ Universidad de Buenos Aires, Facultad de Farmacia y Bioquímica, Departamento de Farmacología, Cátedra de Farmacobotánica y Museo de Farmacobotánica Juan A. Domínguez. E-mails: Imanconatani@ffyb.uba.ar; melinariabis@gmail.com; mlwagner@ffyb.uba.ar 
de julio de ese mismo año por Alexander von Humboldt, la Academia de Ciencias de Francia, con el incentivo de los profesores del Museo de Historia Natural, reclamaron los papeles y las colecciones que el naturalista había generado durante sus largos años de trabajo (Cordier, 1914).

La familia de Bonpland, por intermedio del párroco Jean-Pierre Gay (amigo personal de Bonpland) entregó parte del archivo que conservaban a excepción de papeles personales $\mathrm{y}$ otros que no creyeron convenientes. El intermediario fue el Conde Alfred de Brossard, cónsul de Francia en Asunción del Paraguay (Cordier, 1914; de Gallardo, 1972; Bell, 2010).

Los manuscritos enviados llegaron al Museo de Historia Natural de París en diciembre de 1859. Éstos estaban divididos en: registros de diagnósticos botánicos, listas de semillas, catálogo de geología, notas de viaje, fragmentos descriptivos sin continuación, datos industriales, restos de correspondencias en francés, portugués y español (Bonpland, 1909).

Los libros y papeles con diversas anotaciones, que formaban parte de la documentación generada por el naturalista y que no fueron enviados a Francia son los que atesora hoy el Museo de Farmacobotánica Juan Aníbal Domínguez.

\section{Bonpland y su Archivo}

Con relación a esta documentación y a su herbario particular el mismo Aimé Bonpland, en un fragmento de una carta que envía al astrónomo francés François Arago en 1849, comenta:

"En seguida que regrese á San Borja, donde conservo mis colecciones, y en seguida que la navegación del Uruguay ofrezca la seguridad de la que está privada hace tantos años, enviaré algo á París". "Mi herbario, se compone de más de tres mil plantas que las conservo en buen estado, así como mis manuscritos, da envidia á muchas personas". "Varias veces se me propuso comprarlos y naturalmente he rechazado todos los ofrecimientos..."

(Bonpland, 1909)
Por otro lado, el entonces gobernador de Corrientes Juan Pujol le propuso a Bonpland, en una carta fechada el 18 de octubre de 1854, el cargo de director del Museo de Historia Natural que tenía intenciones de fundar en la provincia. Bonpland aceptó este cargo y en una carta dirigida a Friedrich von Gülich, en uno de sus párrafos, le comentaba que tenía la intención de llevar todos sus manuscritos y colecciones a Santa Ana "donde piensa establecer su cuartel general" (Bonpland, 1909).

\section{Los archivos inéditos}

Luego de la muerte de Bonpland, poco se supo sobre la documentación por él referida y durante muchos años se creyó extraviada en su totalidad. Sin embargo, gran parte de la comunidad científica de distintas épocas tenía noticias de la existencia de un archivo o de notas científicas del naturalista, tal como menciona Eduardo L. Holmberg (1887) en su célebre Viaje a Misiones.

Corría el año 1905 y Eugenio Autran, en ese entonces curador del herbario del Museo de Farmacología de la Facultad de Ciencias Médicas de la Universidad de Buenos Aires², $\mathrm{y}$ el fundador $\mathrm{y}$ director honorario, Juan Aníbal Domínguez, se enteraron por medio de Fernando Schweizer (profesor de Clínica Pediátrica y Puericultura), que un estudiante de apellido Bonpland se encontraba cursando la carrera de Medicina. Ante esta oportunidad, no dudaron en contactarse con este estudiante para averiguar si existía algún parentesco con el naturalista francés. En palabras de Domínguez y Autran:

"Inmediatamente hicimos saber al estudiante nombrado, que deseábamos entrevistarle, y poco tiempo después recibimos en el Museo la visita de un joven de cabello rubio y rostro inteligente, llamado Pompeyo Bonpland. Teníamos en nuestra presencia un nieto del célebre naturalista".

(Domínguez et Autran, 1910)

Pompeyo Bonpland era el nombre del estudiante y les comentó, para su satisfacción,

\footnotetext{
${ }^{2}$ Actual Museo de Farmacobotánica Juan Aníbal Domínguez de la Facultad de Farmacia y Bioquímica de la Universidad de Buenos Aires.
} 
que su familia, que residía en Corrientes, conservaba aún manuscritos y cartas de su abuelo y que intervendría ante ellos para poner a disposición estos documentos.

Algunos meses después se presentó, con una valija repleta de documentos, Amado Bonpland hijo del célebre naturalista $y$ padre del estudiante, y puso a disposición de Domínguez todos los documentos inéditos que conservaban y le dio autorización para que fueran publicados (Domínguez et Autran, 1910).

El 30 de septiembre de 1905 en la revista Caras y Caretas, Holmberg dio por primera vez la noticia del hallazgo de estos documentos, en forma de una crónica detallada (Fig. 1A). En el mismo sentido, el 2 de octubre del mismo año, Autran publicó en el diario francés Le Currier de la Plata, una nota comentando sobre los documentos que donara Bonpland hijo, con el título: "Importante encuentro. Manuscritos de Bonpland. Correspondencia inédita de Humboldt". Desde ese momento, la ciudadanía se puso en conocimiento que parte del archivo del médico y naturalista francés se encontraba depositado en el Museo de Farmacología en Buenos Aires.

En el año 1910, en el XVII Congreso Internacional de los Americanistas, en su sesión de Buenos Aires, Domínguez y Autran comunicaron oficialmente al mundo científico la existencia y el contenido del archivo:

"El archivo Bonpland contiene, además de las 28 cartas inéditas de Humboldt á Bonpland:

Una gran parte de su diario botánico, desde 1849, con la descripción de los números 2450 a 2884 de su herbario.

Numerosos cuadernos manuscritos referentes á sus distintos viajes y sobre la flora y fauna de las regiones argentinas que visitó.

Una serie de cartas de Delille, de Candolle, Mirbell, Hooker, etc.

Cartas del General S. M. Paz relativas á la campaña de Corrientes.

$Y$ numerosas notas sobre la yerba mate, el tabaco, etc."

(Domínguez et Autran, 1910)

Finalizan los autores, esta comunicación, indicando que prontamente se comenzará con la publicación de los documentos del archivo para darlo a conocer a todo mundo científico.

En 1914, Henri Cordier plasmó en "Papiers inédits d' Aimé Bonpland conservés à Buenos Aires", publicado en la serie de Trabajos del Instituto de Botánica y Farmacología ${ }^{3}$, una breve comunicación que había realizado en 1910 al instituto de Francia (Academia de Inscripciones y Bellas Letras) sobre el gran valor que constituía el archivo Bonpland para la ciencia. Este artículo describe brevemente la historia del hallazgo del Archivo, parte de la vida de Bonpland y un escueto catálogo advirtiendo el escaso tiempo empleado para su revisión.

\section{Publicaciones relacionadas al Archivo Bonpland}

Pompeyo Bonpland (1909) presenta su tesis para optar al grado de Doctor en Medicina de la Facultad de Ciencias Médicas de la Universidad de Buenos Aires la cual titula Fragmentos del diario médico de Amado Bonpland. Este trabajo lo realiza bajo la tutela de Juan A. Domínguez y basándose en los papeles inéditos de su abuelo, al cual le rindió un merecido homenaje. Este trabajo puede ser considerado el primero en relación con los papeles donados por la familia Bonpland al Museo y el inicio de una serie de publicaciones relativas a estos documentos, las cuales continúan hasta la actualidad.

Del material contenido en el Archivo histórico del Museo de Farmacobotánica Juan A. Domínguez, se publicaron cuatro tomos en cumplimiento de una resolución de la Facultad de Ciencias Médicas de la Universidad de Buenos Aires y bajo el auspicio del Instituto de Francia.

El primero de ellos, Bonpland (1914) bajo el título "Archives inédites de Aimé Bonpland. Tome I. Lettres inédites de Alexandre de Humboldt. Avec préface de Henri Cordier de L'Institut". El cual lleva el número 31

\footnotetext{
${ }^{3}$ Los Trabajos del Instituto de Botánica y Farmacología son una serie de trabajos de publicación científica periódica realizados por investigadores asociados y del personal técnico de las distintas secciones del, en ese entonces, Instituto de Botánica y Farmacología, hoy denominado Museo de Farmacobotánica Juan Aníbal Domínguez de la Facultad de Farmacia y Bioquímica de la Universidad de Buenos Aires.
} 
de los Trabajos del Instituto de Botánica y Farmacología, y consta de una edición facsimilar de la correspondencia entre Alexander von Humboldt y Aimé Bonpland. Con un prólogo al lector de Domínguez y un prefacio de Cordier (Fig. 1B).

La segunda publicación, Bonpland (1924) apareció bajo el número 42 de los Trabajos del Instituto de Botánica y Farmacología, y se denominó "Archives inédites de Aimé Bonpland. Tomo 2. Journal de Botanique" (Fig. 1B). Este volumen comprende el diario botánico de Bonpland. Domínguez explica, en su prólogo, que este constituiría "...el último de los dos o tres volúmenes que debieron constituirlo, pues se inicia en noviembre de 1849 con la diagnosis $N^{\circ} 2450$, y se detiene con el $N^{\circ} 2884$, en Santa Ana (Corrientes), en diciembre de $1857 . . . "$

Posteriormente, Domínguez (1939a) escribió un pequeño artículo para el Libro Homenaje al Prof. Marian R. Castex con el título "Urquiza y Bonpland. Antecedentes Históricos. La disentería en el ejército Grande en formación, en 1850. Su tratamiento curativo por la 'granadilla': Picramnia Sellowii Planch. v. picraena (Picrasma) palo-amargo (Speg.) Speg. v. Castela Tweedie Planch. (Notas y documentos Inéditos para la Historia de la Medicina Argentina)"4.

El mismo año, Domínguez (1939b) publicó el tomo III del Archivo de Aimé Bonpland denominado: "Documentos para la historia de la República Entrerriana" en la serie II de los Trabajos del Instituto Nacional de Botánica y Farmacología Julio A. Roca ${ }^{5}$. Este volumen cuenta con un prólogo del Doctor Antonio Sagarna y una advertencia de Domínguez.

Un año más tarde (Bonpland, 1940), se publicó el cuarto y último volumen del Archivo Bonpland en el número 2 de la serie II de los Trabajos del Instituto Nacional de Botánica y Farmacología Julio A. Roca con el título "Londres cuartel general europeo. De

4 También publicado en año 1939 como trabajo No59 de la serie de Trabajos del Instituto de Botánica y Farmacología.

${ }^{5}$ Otra de las denominaciones que supo tener el hoy llamado Museo de Farmacobotánica Juan A. Domínguez de la Facultad de Farmacia y Bioquímica de la U.B.A. los patriotas de la emancipación americana" esta publicación cuenta con prólogo del Dr. Guillermo Leguizamón y una advertencia de Domínguez. Con este volumen culminan las publicaciones realizadas por el Dr. Domínguez, en relación con el archivo inédito recibido en 1905 por los herederos de Aimé Bonpland. A pesar de los esfuerzos y los fructíferos trabajos emprendidos no logró concretar el proyecto trazado para la publicación íntegra del Archivo Bonpland, lo cual fue y es una tarea pendiente para las sucesivas generaciones de investigadores que trabajamos en el Museo.

Por último, como corolario, es interesante destacar que Domínguez, por su relación con el Archivo y con la figura de Bonpland y su familia, se constituyó en uno de los mejores conocedores y admiradores de la vida americana de Aimé Bonpland. Publicó una interesante biografía denominada "Aimé Bonpland. Su vida en la América del Sury principalmente en la República Argentina (1817-1858)" (Domínguez, 1929) y posteriormente otra titulada "Biografías. Amado Bonpland" (Domínguez, 1947).

\section{Actualidad}

La voluntad y elección de los descendientes de Aimé Bonpland sobre la responsabilidad y custodia de este acervo está conservada. Sin embargo, a pesar del esfuerzo emprendido por Domínguez y Autran para darlo a conocer por completo al mundo, aún es una tarea inconclusa.

Desde el mes de agosto de 2018 se inició el programa de Recuperación y Puesta en valor del Archivo Bonpland, a través del equipo de trabajo del Museo de Farmacobotánica Juan Aníbal Domínguez.

Estas tareas están dirigidas y gestionadas por uno de los autores, la Lic. Melina Riabis, que es especialista en conservación y restauración, y la Lic. Penélope Mazzoli especialista en archivología. En la actualidad este programa lleva alcanzado con éxito el $20 \%$ del contenido total del Archivo Bonpland.

\section{Programa de Recuperación Patrimonial y Puesta en Valor del Archivo \\ Este programa está implementado en dos etapas principales, aunque en más de una oportunidad ambas ocurren en simultáneo, según las necesidades del material documental.}



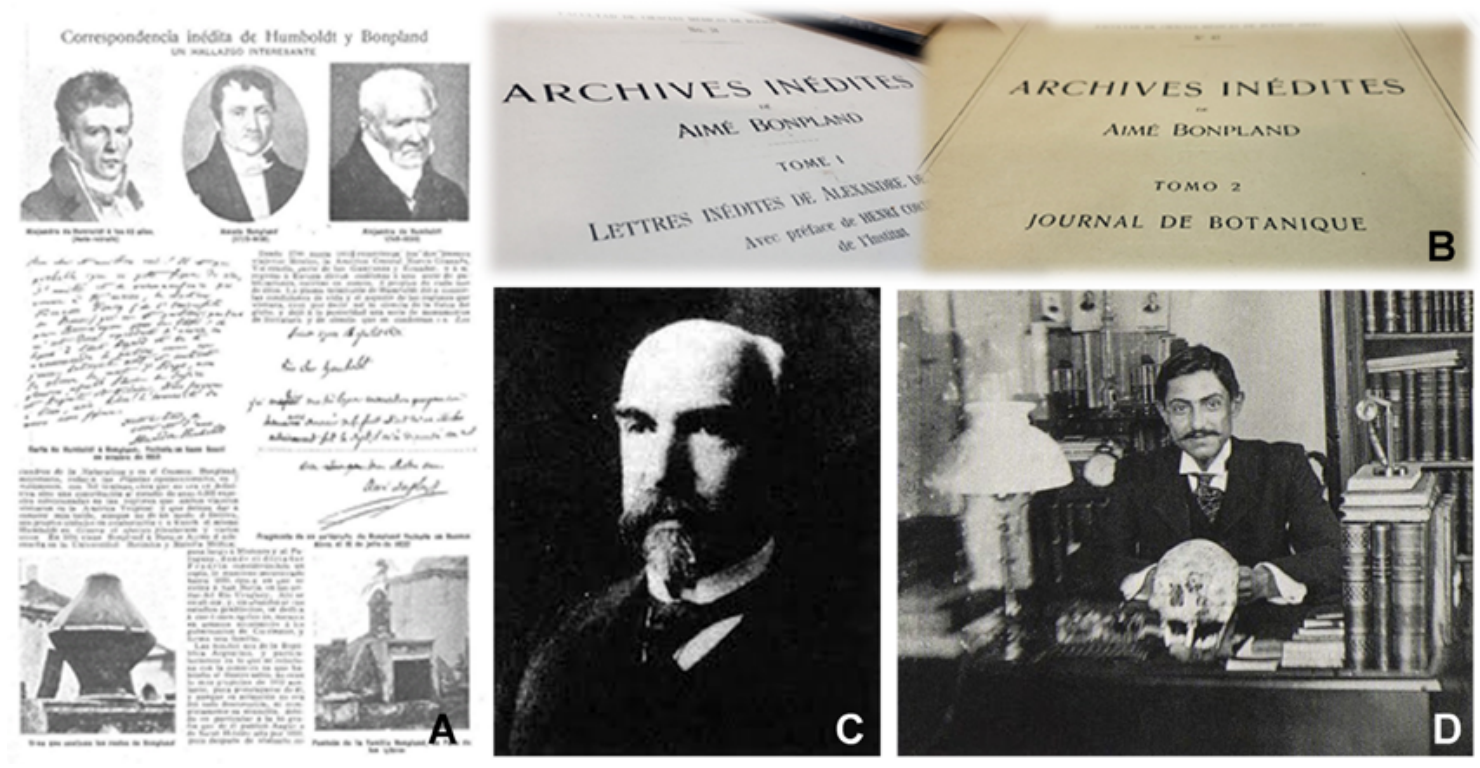

Fig. 1. A: Primera página del artículo publicado por Holmberg (1905) en Caras y Caretas. B: Primeras dos publicaciones del Archivo Bonpland. C: Eugenio Autran (1855-1912). D: Juan Aníbal Domínguez (1876-1946).

Fig. 1. A: First page of the article published by Holmberg (1905) in Caras y Caretas. B: First two publications of the Bonpland Archive. C: Eugene Autran (1855-1912). D: Juan Aníbal Domínguez (1876-1946).

El objetivo final es llegar a una digitalización de acceso público, que no sólo implica la capacidad de acceder a los hechos históricos que constituyen una herramienta clave y fiable de información, sino también generar un fondo coherente, gestionando de forma efectiva este acervo, asegurando la conservación física y permitiendo la sinergia entre distintos fondos documentales existentes.

En cuanto al diagnóstico inicial, podría decirse que la mayoría de los documentos se encuentran estables. Muchos documentos se encuentran guardados en carpetas individuales, las cuales garantizan su buen almacenamiento y correcta manipulación durante la consulta.

Sin embargo, no ocurre en la totalidad de los manuscritos y, menos aún, con los diarios de viaje que presentan un estado crítico de conservación, imposibilitando su manipulación y consulta sin riesgo de pérdida documental. Estos diarios se encuentran en cajas de conservación, pero poseen grandes deterioros en su encuadernación y en los folios internos. En estos últimos se observa principalmente una debilidad estructural, consecuencia de un severo ataque fúngico-bacteriano que tuvo en el pasado, faltantes de material celulósico, oxidación y degradación de las tintas, suciedad, entre otros.

Muchos documentos y los diarios manuscritos no pueden ser sometidos actualmente a la consulta del público, y menos aún a una digitalización, sin antes realizar un responsable diagnóstico completo, una estabilización físico-química y una restauración de sus documentos interiores. En particular, los diarios de viaje deben estar con acceso restringido por su mal estado de conservación y potencial contaminación (Fig. 2A).

\section{Primera etapa: Conservación preventiva}

Las acciones que se llevan a cabo son la limpieza mecánica seca, la estabilización, el control biológico, el cotejo y diagnóstico del estado de conservación de cada uno de los documentos y la realización del nuevo inventario archivístico.

El Archivo Bonpland requiere principalmente, de una limpieza mecánica adecuada, un diagnóstico detallado de su estado de conservación actual y el acondicionamiento del espacio de guarda en el Archivo del Museo (Fig. 2B, C, D). 

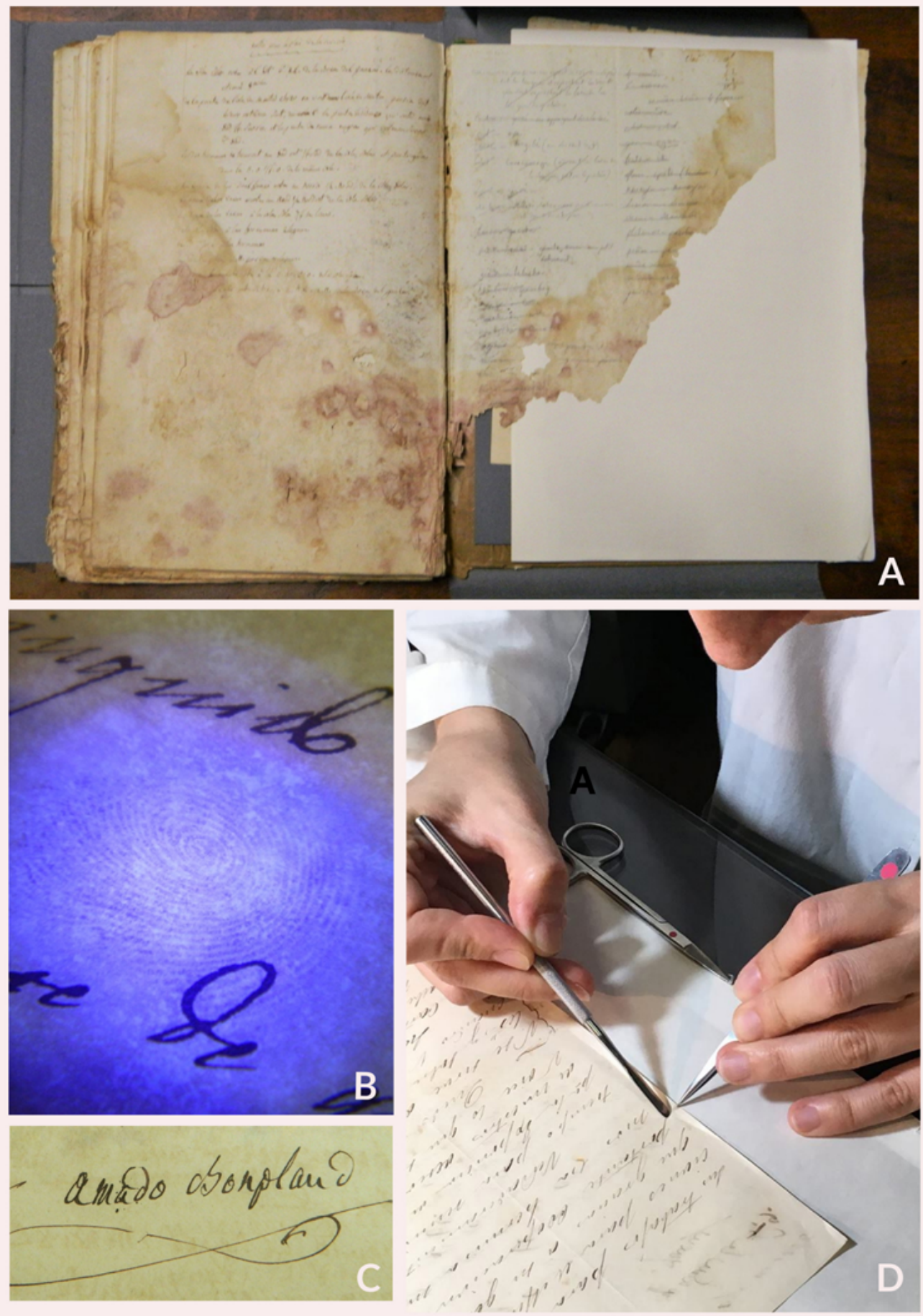

Fig. 2. A: Detalle del Diario de Bonpland 1819-1823 (doc. $N^{\circ} 2044$ ). B: Detalle de huella digital sobre un manuscrito, expuesta bajo lámpara ultravioleta 365 nanómetros de longitud de onda. C: Firma manuscrita de Aimé Bonpland. D: Proceso de restauración sobre las cartas de Bonpland, generando suturas en desgarros.

Fig. 2. A: Detail from Bonpland's Journal 1819-1823 (doc. № 2044). B: Detail of fingerprint on a manuscript, exposed under UV lamp 365 nanometers wavelength. C: Aimé Bonpland's handwritten signature. D: Restoration process on Bonpland letters, generating sutures in ruptures. 
Para llevar a cabo estas acciones, se propuso limpiar cada folio de los documentos con pinceletas suaves, esponjas y gomas aptas bajo parámetros internacionales de conservación. Esta acción permite liberar al papel de suciedad superficial, estabilizar su $\mathrm{pH}$ y realizar un diagnóstico específico y preciso de cada ejemplar en cuanto a sus deterioros físico-químicos.

A su vez, se releva si existen o existieron plagas que dañan al papel de soporte y se registran por escrito y documentan fotográficamente estas cuestiones para futuras restauraciones. También se registran las marcas de agua de los papeles que componen el Archivo, se extrae información de la composición de las tintas y se expone el papel bajo iluminación ultravioleta para observar detalles que no pueden hacerse a simple vista. Esta información se carga y coteja en una base de datos junto al catálogo de la colección.

Se desprende también de este proceder un diagnóstico del espacio de guarda y consulta de este patrimonio, monitoreando el ambiente que lo aloja actualmente. Esto es importante para sentar precedente de la responsabilidad de la custodia de estos volúmenes.

Una vez tratado, limpio y estabilizado el archivo completo, se proyecta acondicionar físicamente los anaqueles y el espacio de guarda definitivo, donde se alojarán nuevamente todos los volúmenes. Además, se está realizando un monitoreo periódico sobre las mediciones de temperatura y humedad relativa, la iluminación y contaminación del ambiente para mantener el ambiente del lugar de guarda, lo más estable posible, dado que no se cuenta con ventilación mecánica artificial.

Esta estabilización permite a futuro, manipular y consultar el material minimizando los riesgos de deterioro de los documentos, amortiguando el impacto medioambiental; por otro lado, dejar acondicionados los volúmenes para una futura restauración y digitalización del archivo, con el objetivo de divulgar su contenido científico, histórico y documental, bajo normas actuales de conservación.

\section{Segunda etapa: Restauración}

En esta segunda etapa se propone, principalmente, la restauración completa de los diarios manuscritos de Bonpland (doc. $\mathrm{n}^{\circ} 2044$ y $\mathrm{n}^{\circ}$ 2046, según catálogo actual) $\mathrm{y}$ de los documentos que en el proceso de diagnóstico pormenorizado se identifiquen con la necesidad de estabilización (ya sea por ruptura, sensibilidad y/o contaminación).

Estas acciones requieren de habilitar dentro del Museo, espacios especiales para la intervención, por resultar procesos que tienen tiempos determinados en que los documentos deben estar inmovilizados y a resguardo de movimientos externos y cambios bruscos.

Las acciones de restauración implican la limpieza puntual de documentos, desinfección, reintegración de zonas faltantes, adhesiones de roturas, desgarros y recuperación de encuadernaciones. Particularmente se proyecta restaurar cada folio que así lo requiera, con materiales vigentes bajo normas internacionales de intervención, según la estabilidad de los soportes y de las tintas que los componen. De este modo se devolverá la flexibilidad al papel, para que sea posible someterlo a la manipulación y la digitalización futura.

$\mathrm{Si}$ bien en este proyecto se establece la necesidad de intervenir los documentos, se prioriza siempre conservar el material original, salvo cuando éste afecte negativamente a el/los documentos. En el caso de retirar materiales originales y reemplazarlos, se almacenan estos retiros por separado para guardar registro histórico y documental (Fig. 3A, B, C).

\section{Digitalización y herramientas de acceso}

En el año 1999, la Fundación Antorchas realizó un proyecto de Conservación preventiva, para el cual se construyeron cajas de guarda aptas, dentro de las cuales se encuentra aún el material, y se confeccionó un escueto catálogo para los documentos.

El nuevo catálogo archivístico, que el Museo propone para el Archivo Bonpland, consiste en crear un inventario itemizado de todos los documentos con mayor detalle, entre ellos: temas, palabras claves, formación de un índice referencial y estado de conservación. Este es un paso esencial en la organización del Archivo y sus herramientas para amplificar su acceso público. De esta manera, es posible buscar material cronológicamente, por temas, o por referencias a ciertas personas, lugares, eventos, entre otros. 

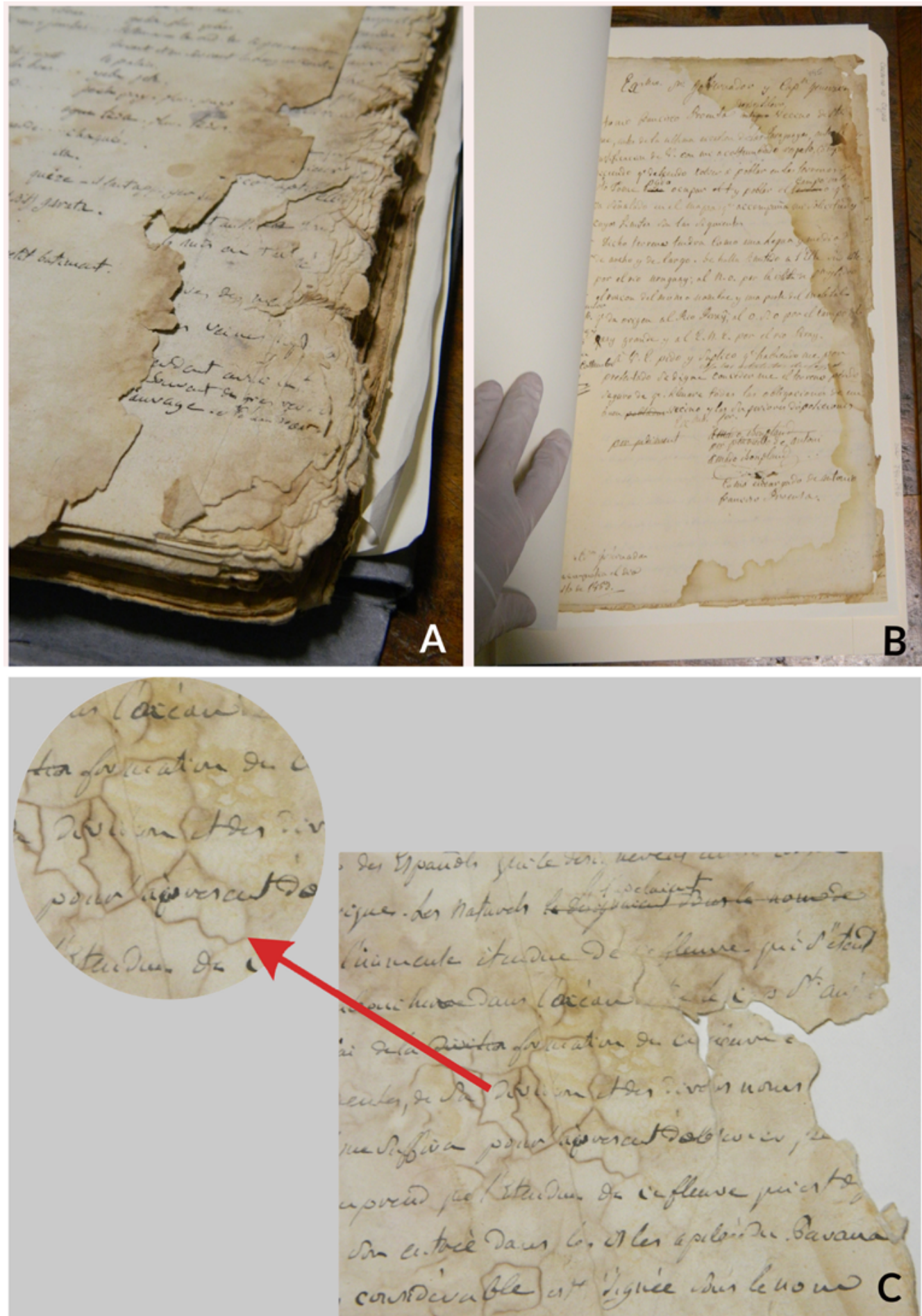

Fig. 3. A y B: Detalle del estado de conservación, friabilidad y manchas del Diario de Bonpland. C: Detalle en aumento de contaminación biológica de uno de los manuscritos.

Fig. 3. A and B: Detail of the state of conservation, friability and stains of the Bonpland's Journal. C: Increasing detail of biological contamination of one of the manuscripts. 
Potencial para la investigación y Actividades de impulso

Conociendo la importancia del material contenido en el Archivo Bonpland, se enumeran algunos posibles proyectos de investigación:

- Desarrollo de la historia política de Corrientes en el siglo XIX.

- Proyectos de agricultura y desarrollo económico.

- El interés científico europeo en Sudamérica.

- El desarrollo de la ciencia a través de cartas con científicos.

- Narrativas de viaje.

El poder acceder al archivo a través de múltiples referencias es sumamente importante para aprovechar el enorme potencial del Archivo Bonpland. Al investigar el diario de viaje de San Borja a Porto Alegre de Aimé Bonpland, por ejemplo, hay referencias a ciertas figuras locales e internacionales con quienes Bonpland tenía relaciones personales y comerciales que podrían rastrearse con mayor facilidad, si fuera posible leer el archivo prestando atención a dichas referencias. Esto permitiría crear un mapa mucho más complejo de la situación política, cultural y económica en un determinado momento y territorio.

\section{Población Beneficiaria del Proyecto}

- Comunidad educativa: docentes y alumnos de la Facultad de Farmacia y Bioquímica de la Universidad de Buenos Aires; visitantes del Archivo: profesores y alumnos de carreras e intereses afines a la botánica, conservación, historia de la ciencia, entre otros.

- Comunidad científica: investigadores vinculados a la historia y a la cultura, material de las ciencias y la educación, historia institucional y académica de la Universidad Buenos Aires, Museos y colecciones universitarias, entre otros.

- Comunidad de los distintos distritos que involucran la presencia del patrimonio sobre Aimé Bonpland: Corrientes, Paraguay, Brasil, Montevideo, París y la ciudad de Buenos Aires.

- Público general: visitantes regulares del Museo y de actividades culturales especiales, como la noche de los museos y la semana de las ciencias, entre otros.
Desde el comienzo de este programa se ha implementado un protocolo de consulta al Archivo, que requiere datos personales y temas de investigación, previo a la consulta de los documentos. Este procedimiento evidencia rutas de acceso al material documental, hecho que brinda mayores herramientas a los parámetros archivísticos implementados dentro del programa y retroalimenta al mismo Archivo.

Durante los años 2018/2019 se ha trabajado en la difusión y comunicación de este proyecto y del Archivo Bonpland, a través de eventos públicos y masivos como son La noche de los Museos y la Semana de las Ciencias, involucrando a los Ministerios de Cultura, de Educación y de Ciencia, Tecnología e Innovación de la Nación. También el Museo de Farmacobotánica participó del II y III Congreso Internacional e Interdisciplinar de Alexander von Humboldt - Aimé Bonpland; $\mathrm{y}$, por último, fue parte del I Encuentro de Archivos Históricos celebrado durante agosto 2019 en la CABA. También se ha hecho mención de este Programa de recuperación y Puesta en valor en la revista The society for the History of Natural History, en una nota reciente para la Televisión Pública, y en las diversas redes sociales vinculadas al custodio de este Archivo, el Museo de Farmacobotánica.

\section{Bibliografía}

BELL, S. (2010). A life in shadow. Aimé Bonpland in Southern South America, 1817-1858. Standford University Press, Standford, California. https://doi.org/10.2307/j.ctvqsdsqm

BONPLAND, P. (1909). Fragmentos del diario médico de Amado Bonpland. Tesis Universidad Nacional de Buenos Aires, Facultad de Ciencias Médicas. Universidad de Buenos Aires. N 2106. Librería y Casa Editora de A. G. Buffarini, Buenos Aires.

BONPLAND, A. (1914). Archives Inédites. Lettres inédites de Alexandre de Humboldt, Tome I. Trabajos del Instituto de Botánica y Farmacología 31. Talleres S. A. Casa Jacobo Pauser, Buenos Aires.

BONPLAND, A. (1924). Archives Inédites. Journal de Botanique, Tomo 2. Trabajos del Instituto de Botánica y Farmacología 42. Talleres S. A. Casa Jacobo Pauser, Buenos Aires. 
BONPLAND, A. (1940). Archivo de Bonpland 4. Londres cuartel general europeo de los patriotas de la emancipación americana. Prólogo del Doctor Guillermo Leguizamón. Trabajos del Instituto de Botánica y Farmacología "Julio A. Roca", Serie II. 2. Imprenta de Pablo Coni, Buenos Aires.

CORDIER, H. M. (1914). Papiers inédits du naturaliste Aimé Bonpland conservés a Buenos Aires. Trabajos del Instituto de Botánica y Farmacología, 30. Jacobo Pauser, Buenos Aires.

DE GALLARDO, T. M. P. (1972). Llegada de los Papeles Inéditos al Museo. Manuscrito inédito.

DOMÍNGUEZ, J. A. \& AUTRAN, E. (1910). Archivos inéditos de Aimé Bonpland existentes en el Instituto de Botánica y Farmacología de la Universidad en la Facultad de Medicina. XVII Congreso Internacional de los Americanistas sesión de Buenos Aires, 16 al 21 de mayo de 1910, pp. 1-2.

DOMÍNGUEZ, J. A. (1929). Aimé Bonpland. Su vida en la América del Sur y principalmente en la República
Argentina (1817-1858). Anales de la Sociedad Científica Argentina 108: 407-435; 497-523.

DOMÍNGUEZ, J. A. (1939a). Urquiza y Bonpland. Antecedentes históricos. La disentería en el Ejército Grande. Trabajos del Instituto de Botánica y Farmacología 59. Imprenta de Pablo Coni, Buenos Aires.

DOMÍNGUEZ, J.A. (1939b). Documentos para la historia de la República Entrerriana del Archivo de Aimé Bonpland. Con prólogo del Doctor Antonio Sagarna. Trabajos del Instituto de Botánica y Farmacología Julio A. Roca, Serie II. 1. Imprenta de Pablo Coni, Buenos Aires.

DOMÍNGUEZ, J. A. (1947). Biografias. Amado Bonpland. Farmalecta 1: 471-474.

HOLMBERG, E. L. (1887). Viaje a Misiones. Boletín de la Academia Nacional de Ciencias de Córdoba, Tomo X. Imprenta de Pablo Coni, Buenos Aires.

HOLMBERG, E. L. (1905). Correspondencia inédita de Humboldt y Bonpland. Un hallazgo interesante. Caras y Caretas 7: 36-37. 